\title{
Exchange and spin relaxation in solid ${ }^{3} \mathrm{He}$
}

\author{
Brian Cowan and Mihail Fardis* \\ Royal Holloway University of London, Egham Hill, Egham, Surrey, TW20 0EX, U.K. \\ E-mail: b.cowanovms.rhbnc.ac.uv
}

Submitted November 26, 1996

\begin{abstract}
Spin relaxation measurements in solid ${ }^{3} \mathrm{He}$ in the temperature region where exchange dominates the behaviour are reviewed and re-analysed. A model is adoptzd which conflates the complex exchange modulation of the dipolar interaction into a single correlation time. This may be regarded as introducing an effective pairwise exchange Hamiltonian. Within the confines of this model new procedures are proposed for obtaining mathematical expressions for the dipolar autocorrelation function and the spectral density functions which determine the relaxation times. By the appropriate treatment of short-time and long-time asymptotic behaviour, together with a method for taking into account the mid-range behaviour, it is possible to fit the experimental data extremely well. The very success of this procedure seems surprising in the light of multiple spin exchange in solid ${ }^{3} \mathrm{He}$. It is an indication that the dominant exchange processes all scaie with density in a similar way. This is supported by path integral Monte Carlo calculations. Some consequencrs and implications of this are discussed.
\end{abstract}

PACS: $67.80 . J d$

\section{Introduction}

Although Thouless [1] pointed out the importance of multiple spin exchange in solid ${ }^{3} \mathrm{He}$ quite some time ago, it was really only with the discovery of the low temperature spin-ordered phases that its real significance was appreciated [2]. Certainly, thermal capacity and magnetization measurements in the paramagnetic phase exhibited some deviations, but the existence of the uudd phase was simply incompatible with pairwise Heisenberg exchange. In retrospect it is surprising that the pairwise exchange model was found to be so successful in the explanation of the higher temperature NMR behaviour of solid ${ }^{3} \mathrm{He}$. It is this question which, ultimately, is addressed in the present paper.

Traditionally NMR has proved a particularly fruitful tool for the study of exchange in solid ${ }^{3} \mathrm{He}$. The use of NMR provides a fairly direct probe of spin behaviour through the measurement of spin susceptibility, spin relaxation times and spin diffusion. In the temperature range around $1 \mathrm{~K}$ the spin relaxation is determined solely by internuclear exchange. Here the temperature is high enough for the exchange *bath to be tightly coupled to the lattice, while it is low enough for there to be negli- gible thermally excited vacancies. Historically this was seen in spin relaxation times which were found to be independent of temperature, but which varied rapidly with density [3].

\section{Spin relaxation - the formalism}

Spin relaxation in solid ${ }^{3} \mathrm{He}$ in the vicinity of $1 \mathrm{~K}$ is caused by the exchange modulation of the internuclear dipolar interaction. The relaxation times $T_{1}$ and $T_{2}$ for this system are given by

$$
\begin{gathered}
\frac{1}{T_{1}}=J_{1}(\omega)+4 J_{2}(2 \omega), \\
\frac{1}{T_{2}}=\frac{3}{2} J_{0}(0)+\frac{5}{2} J_{1}(\omega)+J_{2}(2 \omega)
\end{gathered}
$$

where the spectral density functions $J_{m}(\omega)$ are the Fourier transform of the corresponding dipolar correlation functions $G_{m}(t)$ :

$$
G_{m}(t)=\frac{\operatorname{Tr}\left\{D_{m}(t) D_{m}(0)\right\}}{\hbar^{2} \operatorname{Tr}\left\{I_{z}^{2}\right\}}
$$

and $D_{m}$ are the components of the dipolar Hamiltonian

* Present address: National Centre for Scientific Research «Demokritos*, Athens, Greece. 


$$
D_{m}=\frac{\mu_{0} \hbar^{2} \gamma^{2}}{4 \pi \sqrt{5}} \sum_{i, j}(-1)^{m} \frac{Y_{2}^{-m}\left(\Omega_{i j}\right)}{r_{i j}^{3}} T_{i j}^{m}
$$

The spin part of the interaction is contained in the second order spin tensor operators $T_{i j}^{m}$ :

$$
\begin{gathered}
T_{i j}^{0}=I^{i} \cdot I^{j}-3 I_{z}^{i} I_{z}^{j}, \\
T_{i j}^{1}=\sqrt{3 / 2}\left\{I_{z}^{i} I_{+}^{j}+I_{+}^{i} I_{z}^{j}\right\}=-\left(T T_{i j}^{-1}\right)^{+}, \\
\left.T_{i j}^{2}=-\sqrt{3 / 2} I_{+}^{i} I_{+}^{j}=(T-2)_{i j}^{-}\right)^{+}
\end{gathered}
$$

where the index $m$ denotes the total spin flip induced.

The exchange interaction originates in the large zero-point motion of the ${ }^{3} \mathrm{He}$ atoms which results in the movement of atoms among the lattice sites. The crucial point for multiple spin exchange is that the hard cores of the atoms can favour the coherent exchange of more than two particles [1]. However, since ${ }^{3} \mathrm{He}$ is a spin $1 / 2$ Fermion, an equivalent description of this bodily motion is possible in terms of a spin exchange Hamiltonian. This may be written as

$$
H_{x}=-\sum_{n}(-1)^{n} J_{n} P_{n}^{0}
$$

where $n$ labels the number of particles in each cycle, $J_{n}$ is the exchange frequency for that $n$ particle exchange and $P_{n}^{\sigma}$ is the generator of that permutation of $n$ spins.

In the bcc phase the dominant 2-, 3-, and 4-spin exchange frequencies are of similar order of magnitude $\left(-10^{7} \mathrm{~Hz}\right)$. However since exchange of an odd number of particles is ferromagnetic while exchange of an even number is antiferromagnetic, the resultant exchange can be small; a consequence of inexact cancellation the ferromagnetic and the antiferromagnetic tendencies. Thus we have a frustrated system and there is no a priori reason to expect the observed behaviour to be ferromagnetic or antiferromagnetic. Indeed different properties of such a system can exhibit different characteristics. This is particularly striking in two-dimensional solid ${ }^{3} \mathrm{He}$ films, to be discussed for comparison in Sec. 11.

The lattice structure of the bcc solid means that next-nearest-neighbour exchanges should also be considered. This, together with the variety of multiple spin exchange cycles leads to a complex dynamical system, although there is a simplification which follows since three spin exchanges can be expressed as a superposition of pairwise exchanges. Notwithstanding these complexities, we shall start by considering a simplified and idealized model. The plan of this paper is to study, initially, in detail the consequuences of the pairwise Heisenberg exchange Hamiltonian. It will seen that this model is found to be quite consistent with spin relaxation measurements! Having done we will then attempt to explain the reasons for this apparent success in the light of the current understanding of multiple spin exchange. So for the present the exchange Hamiltonian to be considered is

$$
H_{x}=-2 J \sum_{i<j}^{n n} \mathbf{I}^{i} \cdot \mathbf{p}^{j}
$$

where $i$ and $j$ label the spin sites and the sum is taken over nearest neighbours.

We now have a well-defined problem for solution. Given the expression for the exchange Hamiltonian the time evolution of the dipolar components is given by

$$
D_{m}(t)=\exp \left(\frac{i H_{x} t}{\hbar}\right) D_{m} \exp \left(-\frac{i H_{x} t}{\hbar}\right)
$$

which, from Eq. (2), gives the dipolar autocorrelation function. Fourier transformation then gives the spectral density functions and from Eq. (1) the relaxation times may be found. Unfortunately, as is common in systems of this complexity, the problem does not have an analytic solution. Thus resort must be made to approximate methods. In the following sections we will review the traditional approaches to this, before describing an improved treatment in Sec. 7.

\section{Moment expansions}

At short times the dipolar autocorrelation function may be expanded in powers of time as

$$
G(t)=\frac{1}{3}\left\{M_{2}-\frac{1}{2} M_{4} t^{2}+\ldots\right\}
$$

The coefficients expressed in this way correspond to the conventional moments of the absorption lineshape. The important point about such an expansion, as first noted by Waller [4], is that such moments can be calculated exactly without requiring a complete solution of the problem. Admittedly, evaluation of higher-order moments becomes increasingly difficult, but by diligent application and/or with the aid of a computer meaningful results may be obtained. For our system the second and fourth moments have been evaluated. The second moment is 


$$
M_{2}=\frac{9 C}{20} \frac{\gamma^{2} \hbar^{2}}{a^{6}}
$$

where $a$ is the lattice spacing and $C$ is a constant which depends on the crystal lattice:

$$
\begin{aligned}
& C=12.25 \text { for bcc lattice, } \\
& C=14.45 \text { for hcp lattice. }
\end{aligned}
$$

And the fourth moments are evaluated to be

$$
\begin{aligned}
& M_{4}=22.8 M_{2} J^{2} \text { for bcc lattice, } \\
& M_{4}=42.0 M_{2} J^{2} \text { for hcp lattice. }
\end{aligned}
$$

Note that, as expected, the second moment is independent of motion, here parameterized by $J$, while the fourth moment is strongly dependent on $J$. Armed with very little else, the conventional approach at this point is to choose an appropriate functional form for $G(t)$ which is consistent with these values of $M_{2}$ and $M_{4}$.

\section{Traditional treatment}

Experimentally it is observed, from $T_{1}$ measurements on solid ${ }^{3} \mathrm{He}$, that the spectral density function $J(\omega)$ is reasonably approximated by a Gaussian in the hcp phase and by a decaying exponential in the bcc phase. Thus the hcp correlation function $G(t)$ should be approximately Gaussian, while that for the bcc phase should have a Lorentzian profile. In the conventional treatment of exchange induced relaxation in solid ${ }^{3} \mathrm{He}$ the calculated $M_{2}$ and $M_{4}$ are fitted to these functions. Taking the Fourier transform then yields expressions for the spectral density functions from which the relaxation times may by found as a function of $J$ :

$$
G(t)=\frac{M_{2}}{3} \frac{1}{1+M_{4} t^{2} / 2 M_{2}} \quad G(t)=\frac{M_{2}}{3} \exp \left(-\frac{M_{4}}{2 M_{2}} t^{2}\right) .
$$

But since $M_{4}$ is known in terms of $M_{2}$ and $J$ then

$$
G(t)=\frac{M_{2}}{3} \frac{1}{1+11.4 J^{2} t^{2}} \quad G(t)=\frac{M_{2}}{3} \exp \left(-21 J^{2} t^{2}\right) .
$$

And taking the Fourier transform yields

$$
J(\omega)=\frac{0.31 M_{2}}{J} \exp \left(-\frac{\omega}{3.38 J}\right) \quad J(\omega)=\frac{0.05 M_{2}}{J} \exp \left(-\frac{\omega^{2}}{84 J^{2}}\right)
$$

from which may be found the relaxation times as a function of the exchange frequency $J$ and the Larmor frequency $\omega$.
These spectral density functions make definite predictions about the frequency dependence of the relaxation times, in particular that of $T_{1}$. And in practice these are not quite consistent with the extensive experimental data available. Since these expressions have a single frequency parameter $J$ and the coefficient of $\omega / J$ is fixed by the short time expansion, while the functional form of the high frequency behaviour might be correct it is not possible to ensure precise numerical agreement as there is no free parameter with which to scale the frequency. This casts doubt on the values of $J$ inferred from these analyses. A previous attempt at improving these functional expressions has been made by Guyer et al. [5].

The problem is one of obtaining a plausible approximation to the spectral function on the basis of limited information. Put another way this means making the best possible use of all information available. Thus far we have used knowledge of the short-time behaviour of the autocorrelation function. In the next section we will see that information is available about its long-time behaviour. And in Sec. 6 we will see that something may even be said about intermediate behaviour as well. All information will be drawn together in Sec. 7 where «improved expressions for the spectral density/correlation function will be obtained.

\section{Spin diffusion}

The flip-flop nature of the exchange interaction means that any excess magnetization in part of the specimen will gradually become distributed uniformly. This diffusive process is very much slower than that found in fluids, but it may still be observed by the technique of spin echoes in a field gradient [6].

Since $J$ is the rate at which the $|\uparrow \downarrow\rangle$ spin configuration changes to the $|\uparrow \downarrow\rangle$ configuration, a simple counting argument implies that the order of magnitude of the diffusion coefficient for the magnetization will be $\approx J a^{2}$. Once again, exact calculation of this relation, to find the numerical coefficient is impossible. However a moment method of approximation [7] may be used, and it is actually possible to place bounds on the value of the coefficient [8]. The best estimates are found for the two lattices, to be

$$
\begin{aligned}
& D=0.655 J a^{2} \text { for bcc lattice, } \\
& D=0.860 J a^{2} \text { for hcp lattice. }
\end{aligned}
$$


These relations are well-supported by spin echo measurements [9-11].

Now diffusion is a hydrodynamic process whose validity is limited to the long wavelength, long time limit. At shorter times and distances the precise details of the atomic motion become important. So far as spin relaxation in solid ${ }^{3} \mathrm{He}$ is concerned, this has the implication that both the long-time behaviour of $G(t)$ and the small-frequency behaviour of $J(\omega)$ are determined by the spin diffusion [12]. Thus one has the asymptotic expansion

$$
G(t)-\frac{\hbar^{2} \gamma^{4} \alpha \sqrt{\pi}}{60 \sqrt{2} D^{3 / 2}} t^{-3 / 2}+\ldots
$$

where $\alpha$ is the spin density. This diffusive hydrodynamic behaviour has its corresponding effect in the frequency domain. There one has

$$
J(\omega)=J(0)-\frac{1}{30} \frac{\pi \alpha \hbar^{2} \gamma^{4}}{D^{3 / 2}} \omega^{1 / 2}+\ldots
$$

In both these expressions $D$ may be eliminated in favour of $J$. From Eq. (7) the behaviour of $J(\omega)$ at short frequencies provides another means of measuring the spin diffusion coefficient [12]. This allows the observation of smaller values of $D$, which may be further extended through $T_{1 p}$ measurements. Observe that $J(\omega)$ is not analytic at the origin: This is in contrast to the exponential and Gaussian functions discussed in the previous section.

In Sec. 3 we obtained an expression for the shorttime behaviour of the dipolar autocorrelation function $G(t)$. In this section we have obtained an expression for its long-time behaviour. Any approximation function should satisfy both these conditions.

\section{Universality and the $T_{1}$ minimum}

It is well-known that a minimum in $T_{1}$ occurs as the correlation time of the motion varies at a given Larmor frequency. And the frequency at which the minimum is observed gives an order of magnitude estimate for the motion speed. The minimum in $T_{1}$ is related to the behaviour of the spectral density function in its middle range. In this section we shall formalize these ideas [13]. This will allow us to augment the long- and short-time behaviour of $G(t)$ with further information which any approximation function should respect.

Within the framework of the Heisenberg pairwise exchange model there is a single microscopic time $\tau-J^{-1}$ which characterizes the microscopic dynamical behaviour of the spins. From this a number of general inferences may be made about the relaxation in such cases. This may be seen in the following way. Let us write the autocorrelation function $G(t)$ as the product of its initial value $G(0)$ and a normalized shape function $g$ :

$$
G(t)=G(0) g(t / \tau) .
$$

The shape function $g(t / \tau)$ is unity at $t=0$ and its dimensionless argument $t / \tau$ indicates that $\tau$ is the characteristic time of this system; it is the natural time unit in terms of which the dynamical behaviour of the system scales. Note that for simplicity we are considering a rotationally invariant system so that $G(t)$ need not be encumbered by a spin-flip subscript.

The spectral density function $J(\omega)$ is found from the Fourier transform of $G(t)$ :

$$
J(\omega)=\int_{-\infty}^{\infty} G(0) g(t / \tau) \exp (i \omega t) d t
$$

which, on changing variables of integration through $x=t / \tau$, may be written

$$
J(\omega)=G(0) \tau j(\omega \tau)
$$

where $j(z)$ is the Fourier transform of the shape function $g(x)$.

For simplicity let us start with the simplified expression for $T_{1}$

$$
\frac{1}{T_{1}}=J(\omega)
$$

where the double frequency term has been neglected. It is a straightforward matter to show that this does not detract from the validity of our general conclusions [13], by subsuming the double frequency term into a composite $J(\omega)$ function. The simplified expression for $T_{1}$ is now

$$
\frac{1}{T_{1}}=G(0) \tau j(\omega \tau)
$$

If we divide this expression by $\tau$ or multiply it by $\omega$ then the resulting expressions $1 /\left(T_{1} \tau\right)$ and $\omega / T_{1}$ both depend on $\omega$ and $\tau$ only through the product $\omega \tau:$

$$
\begin{aligned}
& \frac{1}{T_{1} \tau}=G(0) j(\omega \tau), \\
& \frac{\omega}{T_{1}}=G(0) \omega \tau j(\omega \tau) .
\end{aligned}
$$


This means that $T_{1}$ measured at fixed $\tau$, by varying $\omega$, and $T_{1}$ measured at fixed $\omega$, by varying $\tau$ will both be, for a given system, universal functions of $\omega \tau$ [14]. Thus data plotted in these ways will fall on single curves. This will be seen in Sec. 8. For the present, our concern is to discuss the $T_{1}$ minimum.

Generally (except in very unusual circumstances where $j(0)$ diverges) $j(z)$ starts from $j(0)$ and initially it is a slowly decreasing function of its argument, as in Eq. (7). The product $z j(z)$ starts from zero when $z$ is zern, initially growing linearly. However for large $z$ the decay of $j(z)$ will outweigh the linear growth of the $z$ prefactor and the product will decrease. So somewhere in between, when $z$ is of the order of unity, at $z=z^{\prime}$ say, there $z j(z)$ must have a maximum.

Looking at the expression for $T_{1}$ as a function of $\tau$ (at fixed frequency), Eq. (9), we see that the existence of a maximum in $z j(z)$ tells us that there will necessarily be a minimum in $T_{1}$ as the characteristic time is varied. The position of the $T_{1}$ minimum is given by $\omega_{0} \tau=z^{\prime}$ or

$$
\tau_{\min }=z^{\prime} / \omega_{0} \text {. }
$$

Thus when a minimum is observed, to within a constant of the order of unity, the characteristic time of the motion may be estimated by the Larmor period. The value of $T_{1}$ at the minimum may be written, from Eq. (9) as

$$
\frac{1}{T_{1}^{\min }}=\frac{G(0) z^{\prime} j\left(z^{\prime}\right)}{\omega_{0}}
$$

or, since $G(0)=M_{2} / 3$, and denoting the number $z^{\prime} j\left(z^{\prime}\right)$ by $K^{-1}$ :

$$
T_{1}^{\min }=3 K \frac{\omega_{0}}{M_{2}} .
$$

We see that at the minimum the value of $T_{1}$ is proportional to the Larmor frequency. The number $K$ depends on the shape of the autocorrelation function or the spectral density function. Thus, for example, for a Gaussian correlation function $K=0.657$, while for an exponential $K=1$. The value of $K$ for a given system may be found from observations of $T_{1}$ minima over a range of frequencies. This information, also, may be used in the approximation of the spectral density and autocorrelation functions.

\section{Approximating $\mathbf{G}(t)$ and $J(\omega)$}

We now consider specifically the bcc phase of solid ${ }^{3} \mathrm{He}$, and we proceed to the construction of an

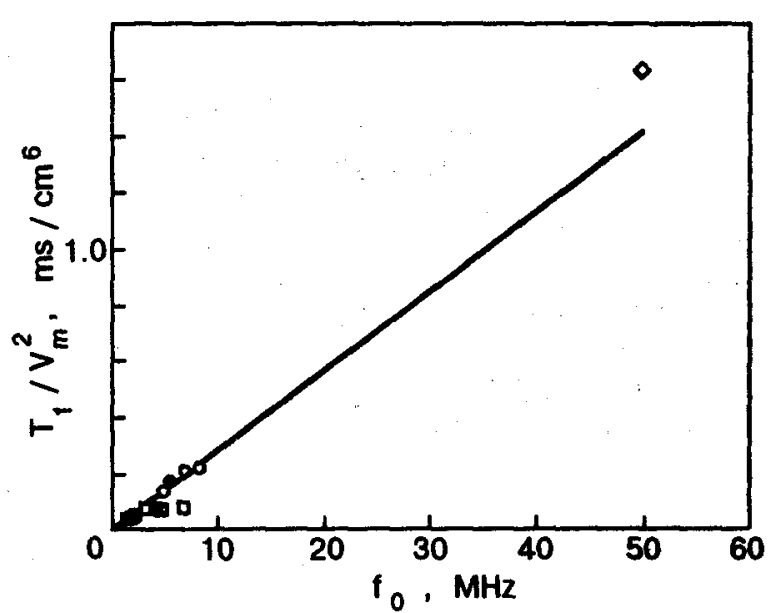

Fig. 1. $T$ minima at different Larmor frequencies for bec ${ }^{3} \mathrm{He}$ $O$ - Richardson, Hunt, and Meyer (1965), Q - Richards, Hatton, and Giffard (1965), - Reich (1963), $\bigcirc$ - Chapellier, Bassou, Devoret, Delrieu, and Sultivan (1985).

approximate spectral density function which is consistent with (i) the short-time microscopic moment expansion of $G(t)$, (ii) the spin diffusion in the hydrodynamic limit, and (iii) the $K$ value characterizing the mid-range region of the $T_{1}$ minima. In order to find the value of $K$ we have collected together $T_{1}$ minimum observations which we have plotted against Larmor frequency in Fig. 1. The $T_{\text {, }}$ data have been divided by the square of the molar volume to remove the small dependence on $M_{2}$ which is also present when the characteristic time is varied by changing the density. The straight line through the data is given by

$$
\frac{T_{1}^{\mathrm{min}}(\mathrm{in} \mathrm{ms})}{V_{m}^{2}\left(\text { in } \mathrm{cm}^{6}\right)}=2.826 \cdot 10^{-2} \frac{\omega_{0}}{2 \pi} \text { (in } \mathrm{MHz} \text { ) . }
$$

This \&fit to the data takes account of the larger experimental error in the data point at $50 \mathrm{MHz}$.

The functional form adopted to approximate $G(t)$ is given by

$$
\frac{G(t)}{G(0)}=\frac{a_{1}}{\left(1+b_{1}^{2} J^{2} t^{2}\right)^{4}}+\frac{a_{2}}{\left(1+b_{2}^{2} J^{2} t^{2}\right)^{3 / 4}}
$$

where the parameters $a_{1}, a_{2}, b_{1}, b_{2}$ are chosen to satisfy the above-mentioned criteria. The second term of this expression gives the correct long-time behaviour, while its Fourier transform leads to the corresponding short-frequency form. The expression for the spectral density approximation function can be written in analytic form as 


$$
\begin{aligned}
& \frac{J(\omega)}{G(0)}=\frac{a_{1} \pi}{96 b_{1} J}\left\{\left(\frac{\omega}{b_{1} J}\right)^{3}+6\left(\frac{\omega}{b_{1} J}\right)^{2}+15\left(\frac{\omega}{b_{1} J}\right)+15\right\} \times \\
& \times \exp \left(-\frac{\omega}{b_{1} J}\right)+\frac{a_{2}}{2^{1 / 4}} \sqrt{\frac{2}{\pi}} \Gamma\left(\frac{1}{4}\right) \frac{1}{b_{2} J}\left(\frac{\omega}{b_{2} J}\right)^{1 / 4} K_{1 / 4}\left(\frac{\omega}{b_{2} J}\right)
\end{aligned}
$$

while the values for the parameters are

$$
\begin{aligned}
& a_{1}=0.840, a_{2}=0.160, \\
& b_{1}=1.768, b_{2}=2.736,
\end{aligned}
$$

$\Gamma$ is the gamma function and $K$ is a Bessel function. We have also included a factor $\xi$ by which the lattice sum value of $M_{2}$ is renormalized as a consequence of the zero-point motion averaging the interparticle spacing $a$ [15]. We find a value of $\xi=0.787$; further discussion of this is deferred until Sec. 9.

The adiabatic part of $T_{2}$ is given in terms of the zero frequency value of $J(\omega)$ :

$$
\frac{1}{T_{2}}=0.270 \frac{M_{2}}{J}
$$

which is the quantitative manifestation of the qualitative result $T_{2}^{-1} \approx M_{2} \tau_{c}$. In other words $\tau_{c}=0.270 / J$; we see that $J^{-1}$ is a measure of the characteristic time for the exchange, but now with a precise numerical multiplier.

\section{Universal plot of relaxation data}

In Fig. 2 we show a plot of all published $T_{1}$ measurements on ${ }^{3} \mathrm{He}$ in the bcc phase presented in sreduced form.

We know, in general, from the discussions of Sec. 6, that for a given system $T_{1} / J$ is a single-valued function of $\omega_{0} / J$. Now since the exchange frequency $J$ is proportional to the frequency at which the $T_{i}$ minimum is observed, it follows that the same universal behaviour will be displayed when plotting $T_{1}$ divided by the frequency of the minimum against Larmor frequency divided by the minimum frequency. This is plotted in Fig. 2, where the $T_{1}$ values have also been divided by the square of the molar volume to account for the variation of $M_{2}$ (and thus $G(0)$ ) with density.

The experimental points are seen to fall very well on a single curve. At higher frequencies there is some discernible deviation from the universal behaviour which we ascribe to differing crystal orientations; the analysis makes the assumption of a

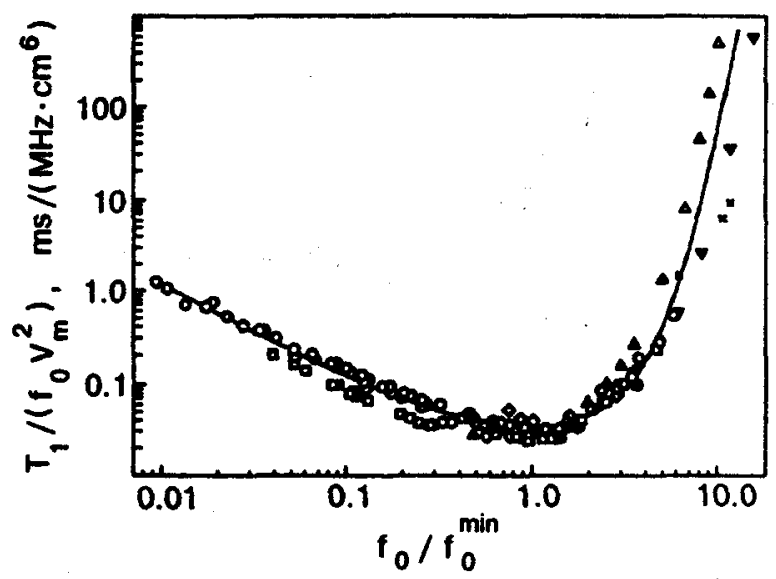

Fig. 2. Reduced plot of $T$, data for bcc ${ }^{3} \mathrm{He}$ together with atheoreticals curve: $\mathrm{O}$ - Richardson, Hunt, and Meyer (1965), 口 - Richards, Hatton, and Giffard (1965), $\Delta$ Thomlinson, Kelley, and Richardson (1972), $\nabla$ - Bernier and Guerrier (1983), 0 - Chapellier, Bassou, Devoret, Delrieu, and Sullivan (1985), - - Reich (1963), X - Kirk and Adams (1972), + - Beal, Giffard, Hatton, Richards, and Richards (1964).

polycrystalline sample, taking averages over crystal orientation. In the low-frequency region it is seen that the data of Richards et al. [3] fall consistently below the other points. It is believed that this is a consequence of inaccuracies in the determination of the molar volume of their crystals.

Having the relaxation data presented in this way facilitates the testing of proposed stheoretical forms for the spectral density function $J(\omega)$. We have the proposed function in Eq. (10). For this function the relation between the exchange frequency and the frequency of the minimum is found to be $\omega_{0}^{\min }=2.42 \mathrm{~J}$. Use of this enables Eq. (10) to be plotted on the graph of experimental data. The solid line in Fig. 2 shows this. We conclude that Eq. (10) does indeed provide a good approximation to the functional form of the spectral density function.

The result of all these considerations is that the exchange frequency can be deduced in a consistent manner from the measurements made at each molar volume. And the best fit to our analysis yields

$$
\frac{J}{2 \pi}=14.08\left(\frac{V_{m}\left(\mathrm{~cm}^{3}\right)}{24}\right)^{n} \mathrm{MHz}
$$

where the exponent $\eta=18.3$. This compares favourably with the results obtained by other means [16]. 


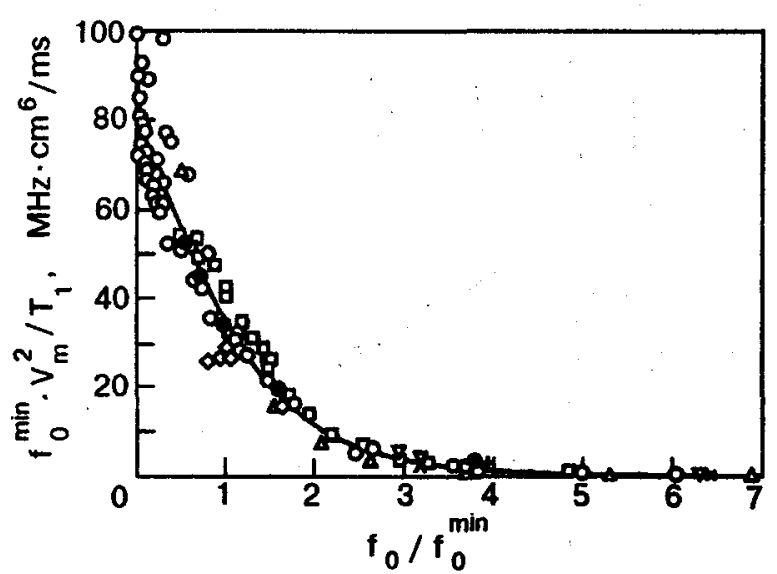

Fig. 3. Second moment sum rule for bec ${ }^{3} \mathrm{He}$. (Key as for Fig. 2).

\section{9. $T_{1}$ sum rules and moments}

Moments were introduced originally in the study of the transverse relaxation. However since, as we have seen in Eq. (5), there is a close connection between the moments and the autocorrelation function $G(t)$, and furthermore since the spin lattice relaxation is related to $J(\omega)$ which is the Fourier transform of $G(t)$, it follows that $T_{1}$ and the moments must be related. We shall investigate such relationships in this section.

Starting from the expression for the dipolar $T_{1}$, Eq. (1a), by a change of variables in the Fouriè integral for the double frequency term, we can write

$$
\frac{1}{T_{1}}=\int_{-\infty}^{\infty}\{G(t)+2 G(t / 2)\} \exp (i \omega t) d t .
$$

Again we have assumed rotational invariance for simplicity but the generalization to distinct $G_{1}(t)$ and $G_{2}(t)$ functions is straightforward. If we now invert this Fourier integral we obtain

$$
G(t)+2 G(t / 2)=\frac{1}{2 \pi} \int_{-\infty}^{\infty} \frac{\exp (-i \omega t)}{T_{1}} d \omega
$$

and then expansion in powers of time leads to

$$
\sum_{n=0}^{\infty} \frac{G^{(n)}(0)}{n !} t^{n}+2 \sum_{n=0}^{\infty} \frac{G^{(n)}(0)}{n !}\left(\frac{t}{2}\right)^{n}=
$$

$$
=\frac{1}{2 \pi} \int_{-\infty}^{\infty} \sum_{n=0}^{\infty} \frac{(-1)^{n} \omega^{n} t^{n}}{n ! T_{1}} d \omega
$$

Equating powers of time then gives, since for even $n$ the integral is symmetric,

$$
\int_{0}^{\infty} \frac{\omega^{n}}{T_{1}} d \omega=(-1)^{n} \pi\left(1+\frac{1}{2^{n-1}}\right) G^{(n)}(0)
$$

which leads to a set of frequency sum rule expressions for $T_{1}$ for increasing $n$. And the sum rules can be expressed in terms of moments, using the relations established in Eq. (5). In this way, for $n=0,2,4$, for example, we obtain

$$
\int_{0}^{\infty} \frac{d \omega}{T_{1}}=\pi M_{2}, \int_{0}^{\infty} \frac{\omega^{2} d \omega}{T_{1}}=\frac{\pi}{2} M_{4}, \int_{0}^{\infty} \frac{\omega^{4} d \omega}{T_{1}} \frac{3 \pi}{8} M_{6}
$$

in the case of rapid motion.

If one has a set of $T_{1}$ data taken over a range of Larmor frequencies then the natural inclination is to interpolate smoothly between the points while extrapolating in an intelligent way beyond the end points. Relations such as the sum rules above provide a test of the validity of such procedures. One can immediately tell if all the area of $J(\omega)$ has been exhausted or if there is some unforeseen behaviour hiding between or beyond the experimental points.

In the present context the $M_{2}$ sum rule may be used for a determination of the renormalization factor $\xi$ alluded to in Sec. 7. The area under the suniversal* plot of $T_{1}$ data in Fig. 3 . gives the renormalized value for $M_{2}$ and the line shown

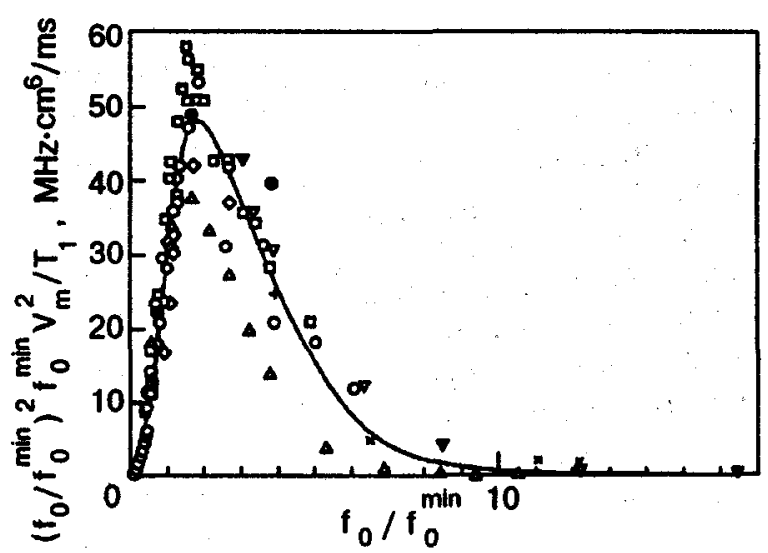

Fig. 4. Fourth moment sum rule for bac ${ }^{3} \mathrm{He}$. (Key as for Fig. 2.) 
corresponds to our spectral density function of Eq. (10), with $\xi=0.787$.

Since $M_{4}=$ const $M_{2} J^{2}$ it follows that $M_{4}$ will be renormalized by the same factor. The area under the *universal p plot of $T_{1}$ data in Fig. 4. gives the renormalized value for $M_{4}$ and the line shown corresponds to our spectral density function of Eq. (10), with the chosen $\xi=0.787$. The fit of the line through the experimental data is not so good in this case, particularly when $f_{0} / f_{0}^{\min }>1$. This plot enhances the high-frequency discrepancies in $T_{1}$; what we see here is a magnified version of the high-frequency deviations of Fig. 2.

\section{Multiple-spin exchange}

The reality of the situation is that pairwise Heisenberg exchange is not adequate to describe the zero point motion in the bcc phase of ${ }^{3} \mathrm{He}$. This becomes evident at lower temperatures. Thermal capacity and magnetic susceptibility may be analyzed on the basis of pairwise exchange, and theoretical expressions for these properties may be obtained as power series in inverse temperature. At lower temperatures, where higher order terms become important, the experimental data are not consistent with the simple Heisenberd model. And at even lower temperatures there is a phase transition to a complex antiferromagnetic spin-ordered phase which definitely requires multiple spin exchange for its explanation.

This being the case it is then paradoxical that the scaling treatment of relaxation works so well, and in particular that the data can be * reduced $\$$ so as to fall 'so well on the universal curve of Fig. 2. The high quality of this reduced data plot implies that so far as NMR relaxation is concerned the system can be understood in terms of a single correlation time characterizing the motion. And in the context of the above discussion the system may be regarded, equivalently, as having an effective pairwise exchange interaction. The effective pairwise exchange frequency will be some functional combination of the frequency of the various interchange processes. Matsumoto et al. [17] have calculated the fourth moment for a restricted subset of two-, three-, and four-particle exchanges. From this we find the effective pairwise exchange frequency determining the NMR relaxation times to be the combination

$$
\begin{aligned}
J_{\text {eff }}^{2}= & J_{n n}^{2}-14 J_{n n} J_{t}+6.7 J_{n n} K_{p}+ \\
& +61 J_{t}^{2}-54 J_{t} K_{p}+18 K_{p}^{2}
\end{aligned}
$$

where $J_{m m}$ is the pairwise exchange frequency, $J_{l}$ is the frequency of the three-particle exchange, and $K_{p}$ is the four-particle exchange frequency for interchanges in a plane.

The reduced data plots incorporate measurements made over a range of molar volumes, where the exchange varies over some two orders of magnitude. The quality of the reduced data plot thi: indicates that the interchange frequencies of the : arious exchange cycles all scale with density in a rather similar manner, as Eq. (11), in fact. Ceperley and Jacucci have investigated this [18] using path-integral Monte Carlo calculations. They obtained the frequencies for a variety of two-, three-, four- and six-spin exchanges (planar 5-spin exchange has also been calculated [19]). And they find that the largest exchange frequencies all scale with density in a similar manner, the exponents being $\eta_{n n}=19.0$, $\eta_{t}=19.8$, and $\eta_{p}=17.6$. There is, however, no $a$ priopi reason to expect these dominant exchange processes to vary with density in the same way: indeed it is a surprise that they do. One is led to wonder, with Gross [20], if multiple spin exchange might be no more than a descriptive construction rather like the planetary epicycles of Ptolomey, and that there may be a more elementary physical description of the atomic motion. Currently there is no satisfactory first-principles theoretical explanation, but it is likely that virtual vacancy-interstitial formation is the fundamental process out of which all exchange cycles are built [19]. The different trajectories taken by the vacancy before reuniting with its interstitial would then correspond to different multiple-spin exchange cycles, the exponent $\eta$ reflecting the probability of creation of the pair. The elucidation of this remains one of the unsolved problems in the theory of solid helium.

\section{Solid ${ }^{3}$ He films}

Further insights into the nature of exchange in bulk solid ${ }^{3} \mathrm{He}$ may be found from a consideration of two-dimensional films of ${ }^{3} \mathrm{He}$. In submonolayer films the observed spin relaxation behaviour is similar to that in three dimensions. The relaxation data may be analysed [21] in a manner similar to that described above. Again, the data may be scaled onto a single curve, implying that here also the various exchange frequencies scale with density in the same way. And when expressed as a function of interparticle spacing the variation in two and three dimensions is similar.

Things are very different, however, when considering multi-layer films of ${ }^{3} \mathrm{He}$. Here the main ex- 
perimental tools have been measurements of spin susceptibility and thermal capacity. Starting with a submonolayer film, as the adsorbate density increases a $2 d$ triangular close packed solid is formed. Increasing the density further, at monolayer completion, there is promotion of atoms to the second layer. This is initially a fluid, but as the density is increased the second layer solidifies. The first layer is paramagnetic and it plays very little part in the observed spin behaviour; this has been confirmed both by analysing the 2-component NMR line profiles and by replacing the first layer with a monolayer of ${ }^{4} \mathrm{He}[22]$.

When it forms, this second solid layer exhibits antiferromagnetic exchange. However, when the density is increased further the third, fluid, layer is formed and this fluid overlayer has a dramatic effect on the second layer, changing it from antiferromagnetic to ferromagnetic. Simultaneous thermal capacity and magnetization measurements have been made [23], which indicate that the changeover becomes manifested in these two properties at different densities. A ferromagnetic thermal capacity can coexist with an antiferromagnetic spin susceptibility! This can be understood in terms of the different combinations of exchange frequencies which enter into the expresions for thermal capacity and spin susceptibility. The fluid overlayer shifts the balance of the different exchange processes. So unlike the bulk solid, in $2 d$ the various exchange frequencies can be varied in different ways; here one has the facility to continuously tune the frustrated spin exchange $[24,25]$. The rich variety of phenomena in bulk solid ${ }^{3} \mathrm{He}$ is thus likely to be exceeded dramatically in solid ${ }^{3} \mathrm{He}$ films.

\section{Acknowledgements}

I am indebted to John Saunders for invaluable discussions in the preparation of this paper. I would also like to thank Bob Richardson and George Pickett for additional information on experimental aspects of some of the data quoted here.

1. D. J. Thouless, Proc. Phys. Soc. 86,893 (1965).

2. M. Roger, J. H. Hetherington, and J. M. Delrieu, Rev. Mod. Phys. 55, I (1983).

3. M. G. Richards, J. Hatton, and R. P. Giffard, Phys. Rev. 139. A.91 (1965)

4. I. Waller, Z. Phys. 79, 370 (1932).

5. R. A. Guyer, R. C. Richardson, and L. I. Zane, Rev. Mod. Phys. 43, 532 (1971).

6. H. C. Torrey, Phys. Rev. 104, 563 (1956).

7. A. G. Redfield, Phys. Rev. 116, 315 (1959).

8. B. P. Cowan, W. J. Mullin, and E. Nelson, J. Low Temp. Phys. 77, 181 (1989).

9. H. A. Reich, Phys. Rev. 129, 630 (1963).

10. J. R. Thompson, E. R. Hunt, and H. Meyer, Phys. Lett. 25A, 313 (1967).

11. B. Eselson, V. Mikheev, and V. Grigor'ev, Sov. J. Low Temp. Phys. 2, 599 (1976).

12. B. P. Cowan and M. Fardis, Phys. Rev. B44, 4304 (1991).

13. B. P. Cowan, L. A. El-Nasr, M. Fardis, and A. Hussain, Phys. Rev. Lett. 58, 2308 (1987).

14. B. P. Cowan, Nuclear Magnetic Resonance and Relaxation. Cambridge University Press (1997).

15. A. Landesman, Ann. de Phys. 8, 53 (1973).

16. M. F. Panczyk and E. D. Adams, Phys. Rev. At, 1356 (1970).

17. K. Matsumoto, T. Abe, and T. Jzuyama, J. Phys. Soc. Jpn. 58, 1149 (1989).

18. D. M. Ceperley and G. Jacucci, Phys. Rev. Lett. 58, 1648 (1988).

19. D. M. Ceperley, Rev. Mod. Phys. 67, 279 (1995)

20. M. Cross, Jpn. J. App. Phys. 26, 1855 (1987).

21. B. P. Cowan, L. A. El-Nasr, M. Fardis, and A. Hussain Phys. Rev. Lett. 58, 2308 (1987).

22. C. P. Lusher, J. Saunders, and B. P. Cowan, Europhys. Lett. 14, 809 (1991).

23. M. Siqueira, J. Nyeki, B. Cowan, and J. Saunders, Phys. Rev. Lett. 76, 1884 (1996).

24. M. Siqueira, J. Nyeki, B. Cowan, and J. Saunders, Czech J. Phys. 46 (1996), in press.

25. M. Siqueira, J. Nyeki, B. Cowan, and J. Saunders, Phys. Rev. Lett. (1996/7), to be published. 